\title{
Evaluation of Post-Partum Anemia and Its Risk Factors and Treatment
}

\author{
Gynecology and Women's Health Care
}

Research Article

Hossam M. Abd-EInaby

Obstetrics and Gynecology Department, Zagazig University Hospital. Zagazig, Egypt

Submitted : December $16^{\text {th }}, 2019$

Correspondence author

Accepted : December 19 $9^{\text {th }}, 2019$

Dr Hossam M. Abd-Elnaby

Published : December $28^{\text {th }}, 2019$

Obstetrics and Gynecology Department

Faculty of Medicine

Zagazig University Hospital

Zagazig

Egypt

E-mail : elnagarw581@gmail.com

\begin{abstract}
Iron deficiency anemia (IDA) in pregnancy, i.e., prepartum anemia, is associated with premature birth, low birth weight, and small for gestational age of the newborn and is furthermore closely associated with the occurrence of anemia after delivery of a child, i.e., in the postpartum period.Post-partum anemia is a common problem throughout the world. through this study aim of the work (frequency of post-partum anemia and risk factors for its development)

The study showed that percentage of antepartum anemia was about $64.3 \%$ this percentage postpartum increased to about $65.4 \%$ and $13.6 \%$ of those with antepartum normal hemoglobin level became anemic. The main causes of postpartum anemia are prepartum iron deficiency anemia in combination with excessive blood losses at delivery. Post-partum anemia is not affected by demographic data, obstetric history, ante-partum u/s finding and obstetric history. Antepartum bleeding was not significantly associated with postpartum anemia. The more the estimated blood loss during labor the more the postpartum anemia. Through follow up of post-partum anemia cases and treated it according type of anemia (oral, intravenous, folate supplementation and blood transfusion). Outcome of treatment of anemia after 8 weeks, $61.02 \%$ was improved and $8.98 \%$ was not improved, due to malabsorption or non-adherence to drug usage.

Conclusion: The main causes of postpartum anemia in this study are prepartum iron deficiency anemia and the amount of blood loss at delivery. this study's results suggest that screening of women with peri delivery $\mathrm{Hb}<10.5 \mathrm{~g} / \mathrm{dL}$ and with increased intrapartum blood loss increase the detection rate of postpartum anemia leading to increased treatment and improved patient care.
\end{abstract}

\section{Introduction}

An adequate iron status is essential for an uncomplicated course of pregnancy, a normal development of the fetus, and maturity of the newborn [1]. Iron deficiency anemia (IDA) in pregnancy, i.e., prepartum anemia, is associated with premature birth, low birth weight, and small for gestational age of the newborn and is furthermore closely associated with the occurrence of anemia after delivery of a child, i.e., in the postpartum period [1].

Postpartum iron deficiency and anemia are associated with an impaired quality of life from a physical and a psychological point of view and constitutes a significant health problem both in developed and developing countries [2]. Anemia after the delivery of a child (postpartum anemia) is a common problem throughout the world [3]. It has been estimated that of the $\sim 500,000$ maternal deaths occurring of anemia in pregnancy prior to delivery (prepartum anemia, which inevitably will be aggravated after delivery due to blood losses [2]. In the Western countries, the prevalence of prepartum anemia in the third trimester is markedly lower in women who have taken iron supplements during pregnancy compared with non-supplemented women. The major causes of postpartum anemia are prepartum iron deficiency/anemia in combination with excessive blood losses at delivery [6-10, 2]. In Europe, in selected series of healthy women after normal delivery, the prevalence of anemia (hemoglobin level $<11 \mathrm{~g} /$ dL) 1 week postpartum is $14 \%$ in women who have taken iron supplements in pregnancy vs. $24 \%$ in non-supplemented women. In developing countries, the prevalence of postpartum anemia is considerably higher, in the range of $70 \%-80 \%$ [10]. each year on a global scale in association with delivery, $20 \%$ are caused by peripartum hemorrhage and anemia $[4,5]$. Postpartum anemia is closely connected with the presence 
The post-partum period is defined as the period during which conditions return to the non-pregnant states [6]. Some studies have focused on iron administration to prevent anemia and the results obtained during pregnancy [7]. However, in Japan, it is conventionally reported that anemia will be alleviated by the 30th day post-partum recommends dietary therapy rather than prescribing iron tablets for p.o. administration when the hemoglobin level is between 9 and $11 \mathrm{~g} / \mathrm{dL}$ and mean corpuscular volume (MCV) is between 85 and $100 \mu \mathrm{m} 3$. If anemia is not alleviated by the 30th day post-partum, this will impede a full recovery from the delivery and will complicate childcare, resulting in increases in fatigue [8].

\section{Aim}

The aim of the study is to know the percentage of post-partum anemia and what are the risk factors for its development.

\section{Patients and Methods:}

- This study was a prospective cross section observational study

- This research was done according to Helsinki declaration for research in human being, an informed written consent was taken from all participants before recruitment in the study, after proper counseling and a very clear explanation of the purpose.

Conducted at Obstetrics and Gynecology department of Zagazig University during the period (from September 2017 to January 2018).

- Population in patients around 6000 in one year delivered.

- Confidence interval (CI):95\%.

- Power: $80 \%$.

- Frequency:20\%.

- so, sample size 361 case.

\section{The Inclusion Criteria:}

All cases who delivered vaginally in Zagazig University Hospital. During the period (from September 2017 to January 2018).

\section{The exclusion criteria:}

- Patient who had medical or surgical disorder affecting hemoglobin level: including: fever, infection, diabetes mellitus, liver disease, renal disease, tuberculosis, cardiovascular disease, gastrointestinal symptoms/disease blood disease or cancer.

- Peripartum blood transfusion.

- Multiple gestation

- Cesarean section

- Vaginal birth after cesarean section

- Hypertensive disorder

All patients were undergone the following

Consent was taken from all patients

- Complete history taking, history of iron supplementation during pregnancy.

History of antepartum bleeding.

Complete clinical examination.

Complete blood picture $(\mathrm{CBC})$ test was done and recorded for every patient enrolled in the study within 2 weeks before delivery and within two weeks after delivery, $3 \mathrm{ml}$ of blood was withdrawn by venipuncture into dry plastic tube containing EDETA solution. Hemoglobin (HB) concentration, hematocrit value $(\mathrm{HCV})$, and other indices (mean corpuscular hemoglobin or $\mathrm{MCH}$, mean corpuscular hemoglobin concentration or $\mathrm{MCHC}$, and mean corpuscular volume or MCV) was tabulated and recorded for every patient in the study.

Cases of PPA diagnosed at Level of hemoglobin concentration $<10 \mathrm{gm} / \mathrm{dl}$ ). Case with PPA and (severe anemia below $8 \mathrm{gm} / \mathrm{dl}$ ) was compared with cases without PPA as regarding:

- HB before delivery.

- Antepartum hemorrhage.

- Estimated blood loss during labor.

Cases who need treatment were given oral or intravenous iron., plus folic acid and vitamin B12 according to the type of anemia and cases with hemoglobin less than $6 \mathrm{gm} / \mathrm{dl}$ were given blood transfusion. (Treatment with iron supplements was performed according to the following protocol; women with $\mathrm{Hb}>9 \mathrm{~g} / \mathrm{dL}$ were treated with oral iron supplements (usually those that were taken during pregnancy), women with $\mathrm{Hb} \leq 9 \mathrm{~g} / \mathrm{dL}$ received in addition one dose of I.V. $500 \mathrm{mg}$ iron sucrose. Women with $\mathrm{Hb}$ between 7 and $7.9 \mathrm{~g} / \mathrm{dL}$ with anemia-related symptoms or with $\mathrm{Hb}<7 \mathrm{~g} / \mathrm{dL}$ regardless of symptoms also received a transfusion of packed red blood cells).

- Follow up CBC was done 8 weeks after delivery

\section{Statistical Analysis}

All data were collected, tabulated and statistically analyzed using SPSS 20.0 for windows (SPSS Inc., Chicago, IL, USA) and MedCalc 13 for windows (MedCalc Software bvba, Ostend, Belgium). Quantitative data were expressed as the mean $\pm \mathrm{SD} \&$ median (range), and qualitative data were expressed as absolute frequencies (number) \& relative frequencies (percentage).

\section{Results}

Initially, 409 included in this study. 34 patients refused Participation, 14 missed follow up lastly 361 involved in the study. Sample size 361 case the study showed postpartum anemia 236 case percentage of postpartum anemia is $65.4 \%$. The study showed that percentage of antepartum anemia was about $64.3 \%$ this percentage postpartum increased to about $65.4 \%$ and $13.6 \%$ of those with antepartum normal hemoglobin level became anemic.

\section{Types of postpartum anemia}

- mild normocytic normochromic anemia(HB $10 \mathrm{~g} / \mathrm{dl})(100$ case).

- mild microcytic hypochromic anemia (68 case). 
- moderate normocytic normochromic anemia(HB 8-9.5 g/ dl) (33 case).

- moderate microcytic hypochromic anemia (27 case).

- severe normocytic normochromic anemia $(\mathrm{HB}<8 \mathrm{~g} / \mathrm{dl})(3$ case).

- $\quad$ severe microcytic hypochromic anemia (5 case).

The main causes of postpartum anemia are prepartum iron deficiency/anemia and intrapartum blood loss at delivery. Postpartum anemia is not affected by demographic data, obstetric history, ante-partum u/s finding. Antepartum hemorrhage was not significantly associated with postpartum anemia. The more the estimated blood loss during labor the more the postpartum anemia .Through follow up of post-partum anemia cases and treated it according type of anemia (oral, intravenous, folate supplementation and blood transfusion) Outcome of treatment of anemia after 8 weeks $61.02 \%$ is improved $\& 38.98 \%$ is not improved.

Table 1: Prevalence of postpartum anemia in the studied pregnant women.

\begin{tabular}{|l|l|l|}
\hline \multirow{2}{*}{ Postpartum anemia } & \multicolumn{2}{|l|}{ Total (N=361) } \\
\cline { 2 - 3 } & No & $\%$ \\
\hline Absent & 125 & $34.6 \%$ \\
\hline Present & 236 & $65.4 \%$ \\
\hline $\begin{array}{l}\text { Mild normocytic } \\
\text { normochromic } \\
\text { (HB 9-10 g/dl) }\end{array}$ & 100 & $42.37 \%$ \\
\hline $\begin{array}{l}\text { Mild microcytic } \\
\text { hypochromic }\end{array}$ & 68 & $28.81 \%$ \\
\hline $\begin{array}{l}\text { Moderate normocytic } \\
\text { normochromic } \\
\text { (HB 8-9 g/dl) }\end{array}$ & 33 & $13.98 \%$ \\
\hline $\begin{array}{l}\text { Moderate microcytic } \\
\text { hypochromic }\end{array}$ & 27 & $11.44 \%$ \\
\hline $\begin{array}{l}\text { Severe normocytic } \\
\text { normochromic } \\
\text { (HB }<8 \text { g/dl) }\end{array}$ & 3 & $1.27 \%$ \\
\hline $\begin{array}{l}\text { Severe microcytic } \\
\text { hypochromic }\end{array}$ & 5 & $2.12 \%$ \\
\hline
\end{tabular}

Table 2: Prevalence of antepartum anemia in the studied pregnant women.

\begin{tabular}{|l|l|l|}
\hline \multirow{2}{*}{ Antepartum anemia } & \multicolumn{2}{l|}{ Total (N=361) } \\
\cline { 2 - 3 } & No & $\%$ \\
\hline Absent & 129 & $35.7 \%$ \\
\hline Present & 232 & $64.3 \%$ \\
\hline $\begin{array}{l}\text { Mild normocytic } \\
\text { normochromic }\end{array}$ & 105 & $45.26 \%$ \\
\hline $\begin{array}{l}\text { Mild microcytic } \\
\text { hypochromic }\end{array}$ & 77 & $33.19 \%$ \\
\hline $\begin{array}{l}\text { Moderate normocytic } \\
\text { normochromic }\end{array}$ & 20 & $8.62 \%$ \\
\hline $\begin{array}{l}\text { Moderate microcytic } \\
\text { hypochromic }\end{array}$ & 20 & $8.62 \%$ \\
\hline $\begin{array}{l}\text { Severe normocytic } \\
\text { normochromic }\end{array}$ & 5 & $2.16 \%$ \\
\hline $\begin{array}{l}\text { Severe microcytic } \\
\text { hypochromic }\end{array}$ & 5 & $2.16 \%$ \\
\hline
\end{tabular}

Table 3: Relation between antepartum anemia and postpartum anemia in the studied pregnant women.

\begin{tabular}{|l|l|l|l|l|}
\hline \multicolumn{2}{|c|}{} & \multicolumn{2}{|l|}{ Postpartum anemia } & \multirow{2}{*}{ Total } \\
\cline { 3 - 5 } \multicolumn{2}{|c|}{} & Absent & Present & \\
\hline $\begin{array}{l}\text { Antepartum } \\
\text { anemia }\end{array}$ & Absent & $\begin{array}{l}80 \\
(22.2 \%)\end{array}$ & $\begin{array}{l}49 \\
(13.6 \%)\end{array}$ & $\begin{array}{l}129 \\
(35.7 \%)\end{array}$ \\
\cline { 2 - 5 } & Present & $\begin{array}{l}45 \\
(12.5 \%)\end{array}$ & $\begin{array}{l}187 \\
(51.8 \%)\end{array}$ & $\begin{array}{l}232 \\
(64.3 \%)\end{array}$ \\
\hline Total & $\begin{array}{l}125 \\
(34.6 \%)\end{array}$ & $\begin{array}{l}236 \\
(65.4 \%)\end{array}$ & $\begin{array}{l}361 \\
(100 \%)\end{array}$ \\
\hline
\end{tabular}


Table 4: Relation between type of antepartum anemia and postpartum anemia in the studied pregnant women.

\begin{tabular}{|c|c|c|c|c|c|c|c|c|c|}
\hline & & \multicolumn{7}{|c|}{ Postpartum anemia } & \multirow[t]{2}{*}{ Total } \\
\hline & & $\begin{array}{c}\text { No } \\
\text { anemia }\end{array}$ & $\begin{array}{l}\text { Mild } \\
\text { NN }\end{array}$ & $\begin{array}{l}\text { Mild } \\
\text { MH }\end{array}$ & $\begin{array}{l}\text { Mod. } \\
\text { NN }\end{array}$ & $\begin{array}{l}\text { Mod. } \\
\text { MH }\end{array}$ & $\begin{array}{l}\text { Sev. } \\
\text { NN }\end{array}$ & $\begin{array}{l}\text { Sev. } \\
\text { MH }\end{array}$ & \\
\hline \multirow[t]{7}{*}{$\begin{array}{l}\text { Antepartum } \\
\text { anemia }\end{array}$} & $\begin{array}{c}\text { No } \\
\text { Anemia }\end{array}$ & $\begin{array}{c}80 \\
(22.2 \%)\end{array}$ & $\begin{array}{c}28 \\
(7.8 \%)\end{array}$ & $\begin{array}{c}14 \\
(3.9 \%)\end{array}$ & $\begin{array}{c}5 \\
(1.4 \%)\end{array}$ & $\begin{array}{c}1 \\
(0.3 \%)\end{array}$ & $\begin{array}{c}0 \\
(0 \%)\end{array}$ & $\begin{array}{c}1 \\
(0.3 \%)\end{array}$ & $\begin{array}{c}129 \\
(35.7 \%)\end{array}$ \\
\hline & $\begin{array}{l}\text { Mild } \\
\text { NN }\end{array}$ & $\begin{array}{c}23 \\
(6.4 \%)\end{array}$ & $\begin{array}{c}54 \\
(15 \%)\end{array}$ & $\begin{array}{c}9 \\
(2.5 \%)\end{array}$ & $\begin{array}{c}9 \\
(2.5 \%)\end{array}$ & $\begin{array}{c}7 \\
(1.9 \%)\end{array}$ & $\begin{array}{c}1 \\
(0.3 \%)\end{array}$ & $\begin{array}{c}2 \\
(0.6 \%)\end{array}$ & $\begin{array}{c}105 \\
(45.26 \%)\end{array}$ \\
\hline & $\begin{array}{l}\text { Mild } \\
\text { MH }\end{array}$ & $\begin{array}{c}15 \\
(4.2 \%)\end{array}$ & $\begin{array}{c}13 \\
(3.6 \%)\end{array}$ & $\begin{array}{c}37 \\
(10.2 \%)\end{array}$ & $\begin{array}{c}5 \\
(1.4 \%)\end{array}$ & $\begin{array}{c}7 \\
(1.9 \%)\end{array}$ & $\begin{array}{c}0 \\
(0 \%)\end{array}$ & $\begin{array}{c}0 \\
(0 \%)\end{array}$ & $\begin{array}{c}77 \\
(33.19 \%)\end{array}$ \\
\hline & $\begin{array}{l}\text { Mod. } \\
\text { NN }\end{array}$ & $\begin{array}{c}3 \\
(0.8 \%)\end{array}$ & $\begin{array}{c}3 \\
(0.8 \%)\end{array}$ & $\begin{array}{c}4 \\
(1.1 \%)\end{array}$ & $\begin{array}{c}9 \\
(2.5 \%)\end{array}$ & $\begin{array}{c}1 \\
(0.3 \%)\end{array}$ & $\begin{array}{c}0 \\
(0 \%)\end{array}$ & $\begin{array}{c}0 \\
(0 \%)\end{array}$ & $\begin{array}{c}20 \\
(8.62 \%)\end{array}$ \\
\hline & $\begin{array}{l}\text { Mod. } \\
\text { MH }\end{array}$ & $\begin{array}{c}3 \\
(0.8 \%)\end{array}$ & $\begin{array}{c}1 \\
(0.3 \%)\end{array}$ & $\begin{array}{c}4 \\
(1.1 \%)\end{array}$ & $\begin{array}{c}3 \\
(0.8 \%)\end{array}$ & $\begin{array}{c}9 \\
(2.5 \%)\end{array}$ & $\begin{array}{c}0 \\
(0 \%)\end{array}$ & $\begin{array}{c}0 \\
(0 \%)\end{array}$ & $\begin{array}{c}20 \\
(8.62 \%)\end{array}$ \\
\hline & $\begin{array}{l}\text { Sev. } \\
\text { NN }\end{array}$ & $\begin{array}{c}0 \\
(0 \%)\end{array}$ & $\begin{array}{c}1 \\
(0.3 \%) \\
\end{array}$ & $\begin{array}{c}0 \\
(0 \%)\end{array}$ & $\begin{array}{c}1 \\
(0.3 \%) \\
\end{array}$ & $\begin{array}{c}1 \\
(0.3 \%)\end{array}$ & $\begin{array}{c}2 \\
(0.6 \%) \\
\end{array}$ & $\begin{array}{c}0 \\
(0 \%) \\
\end{array}$ & $\begin{array}{c}5 \\
(2.16 \%)\end{array}$ \\
\hline & $\begin{array}{l}\text { Sev. } \\
\text { MH }\end{array}$ & $\begin{array}{c}1 \\
(0.3 \%)\end{array}$ & $\begin{array}{c}0 \\
(0 \%)\end{array}$ & $\begin{array}{c}0 \\
(0 \%)\end{array}$ & $\begin{array}{c}1 \\
(0.3 \%)\end{array}$ & $\begin{array}{c}1 \\
(0.3 \%)\end{array}$ & $\begin{array}{c}0 \\
(0 \%)\end{array}$ & $\begin{array}{c}2 \\
(0.6 \%)\end{array}$ & $\begin{array}{c}5 \\
(2.16 \%)\end{array}$ \\
\hline \multicolumn{2}{|c|}{ Total } & $\begin{array}{c}125 \\
(34.6 \%)\end{array}$ & $\begin{array}{c}100 \\
(42.37 \%)\end{array}$ & $\begin{array}{c}68 \\
(28.81 \%)\end{array}$ & $\begin{array}{c}33 \\
(13.98 \%)\end{array}$ & $\begin{array}{c}27 \\
(11.44 \%)\end{array}$ & $\begin{array}{c}3 \\
(1.27 \%)\end{array}$ & $\begin{array}{c}5 \\
(2.12 \%)\end{array}$ & $\begin{array}{c}361 \\
(100 \%)\end{array}$ \\
\hline
\end{tabular}


Table 5: Relation between demographic data and postpartum anemia.

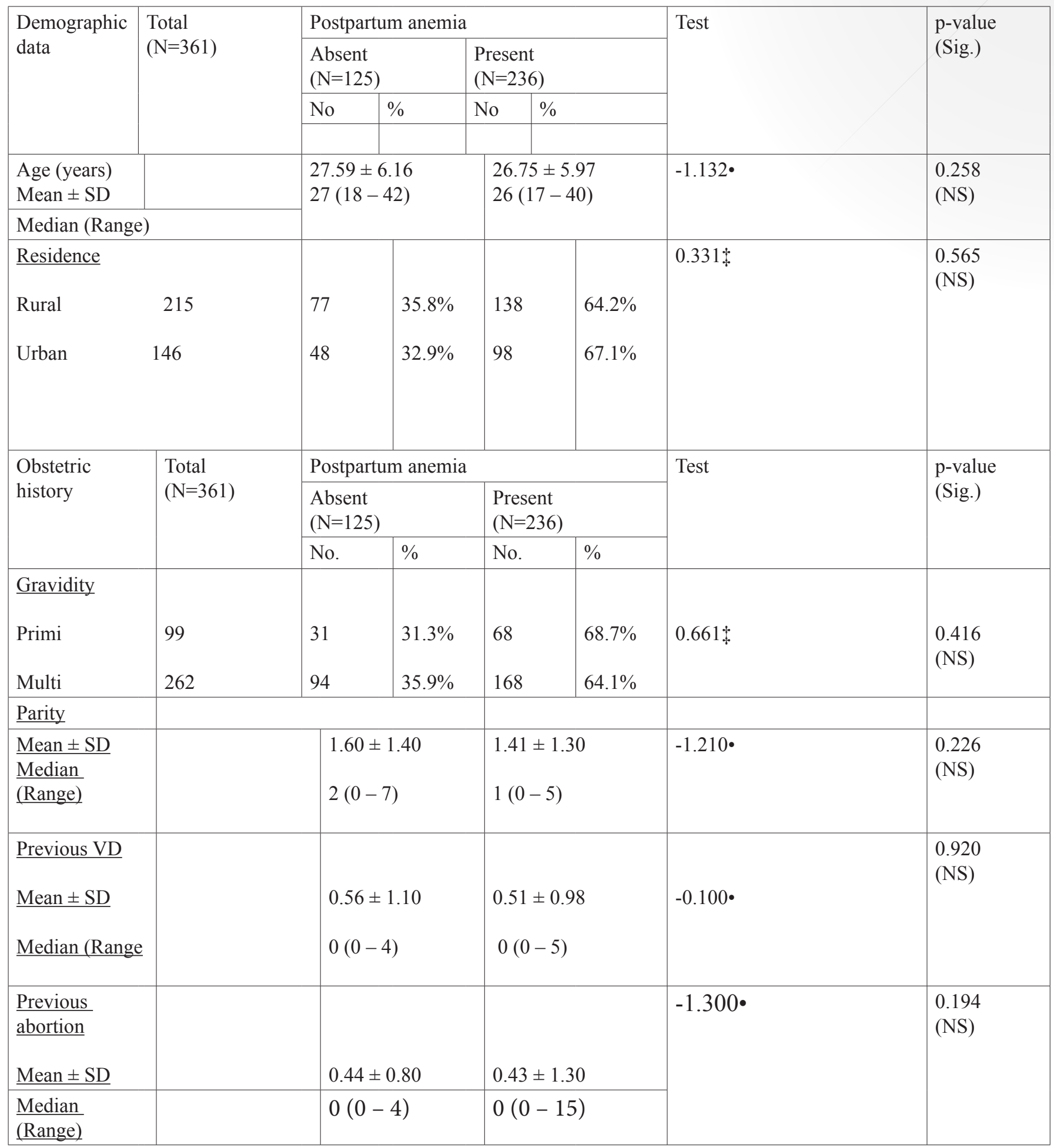


Table 6: Relation between ante-partum U/S , obstetric history and postpartum anemia.

\begin{tabular}{|c|c|c|c|c|c|c|c|}
\hline \multirow[t]{3}{*}{ Ante-partum U/S } & \multirow{3}{*}{\begin{tabular}{|l} 
Total \\
$(\mathrm{N}=361)$
\end{tabular}} & \multicolumn{4}{|c|}{ Postpartum anemia } & \multirow[t]{3}{*}{ Test: } & \multirow{3}{*}{$\begin{array}{l}\text { p-value } \\
\text { (Sig.) }\end{array}$} \\
\hline & & \multicolumn{2}{|c|}{ Absent $(\mathrm{N}=125)$} & \multicolumn{2}{|c|}{ Present $(\mathrm{N}=236)$} & & \\
\hline & & No. & $\%$ & No. & $\%$ & & \\
\hline $\begin{array}{l}\text { Viability of fetus } \\
\text { Viable } \\
\text { IUFD }\end{array}$ & $\begin{array}{l}346 \\
15\end{array}$ & $\begin{array}{l}120 \\
5\end{array}$ & $\begin{array}{l}34.7 \% \\
33.3 \%\end{array}$ & $\begin{array}{l}226 \\
10\end{array}$ & $\begin{array}{l}65.3 \% \\
66.7 \% \\
\end{array}$ & 0.012 & $\begin{array}{l}0.914 \\
(\mathrm{NS})\end{array}$ \\
\hline $\begin{array}{l}\text { Sex of baby } \\
\text { Male } \\
\text { Female }\end{array}$ & $\begin{array}{l}180 \\
181\end{array}$ & $\begin{array}{l}53 \\
72\end{array}$ & $\begin{array}{l}29.4 \% \\
39.8 \%\end{array}$ & $\begin{array}{l}127 \\
109\end{array}$ & $\begin{array}{l}70.6 \% \\
60.2 \%\end{array}$ & 4.258 & $\begin{array}{l}0.039 \\
(\mathrm{NS})\end{array}$ \\
\hline $\begin{array}{l}\frac{\text { Fetal weight }}{2500-3000 \mathrm{mg}} \\
3000-3200 \\
\text { Less than } 2500\end{array}$ & $\begin{array}{l}312 \\
10 \\
2 \\
\end{array}$ & $\begin{array}{l}105 \\
5 \\
1 \\
\end{array}$ & $\begin{array}{l}33.7 \% \\
50 \% \\
50 \% \\
\end{array}$ & $\begin{array}{l}207 \\
5 \\
1 \\
\end{array}$ & $\begin{array}{l}66.3 \% \\
50 \% \\
50 \% \\
\end{array}$ & 1.552 & $\begin{array}{l}0.670 \\
(\mathrm{NS})\end{array}$ \\
\hline $\begin{array}{l}\text { Amniotic fluid } \\
\text { Normal } \\
\text { Oligohydramnios } \\
\text { Polyhydramnios } \\
\text { Anhydraminos }\end{array}$ & \begin{tabular}{|l|}
330 \\
26 \\
4 \\
1 \\
\end{tabular} & $\begin{array}{l}110 \\
10 \\
4 \\
1\end{array}$ & $\begin{array}{l}33.3 \% \\
38.5 \% \\
100 \% \\
100 \% \\
\end{array}$ & $\begin{array}{l}220 \\
16 \\
0 \\
0\end{array}$ & $\begin{array}{l}66.7 \% \\
61.5 \% \\
0 \% \\
0 \% \\
\end{array}$ & 9.853 & $\begin{array}{l}0.020 \\
(\mathrm{NS})\end{array}$ \\
\hline \multirow[t]{3}{*}{ Partum history } & \multirow{3}{*}{$\begin{array}{l}\text { Total } \\
(\mathrm{N}=361)\end{array}$} & \multicolumn{4}{|c|}{ Postpartum anemia } & \multirow[t]{3}{*}{ Test } & \multirow{3}{*}{$\begin{array}{l}\text { p-value } \\
\text { (Sig.) }\end{array}$} \\
\hline & & \multicolumn{2}{|c|}{ Absent $(\mathrm{N}=125)$} & \multicolumn{2}{|c|}{ Present $(\mathrm{N}=236)$} & & \\
\hline & & No & $\%$ & No & $\%$ & & \\
\hline $\begin{array}{l}\frac{\text { Gestational age }}{\text { (weeks) }} \\
\text { Mean } \pm \text { SD } \\
\text { Median (Range) }\end{array}$ & & \multicolumn{2}{|c|}{$\begin{array}{l}37.04 \pm 2.72 \\
38(27-43)\end{array}$} & \multicolumn{2}{|c|}{$\begin{array}{l}36.77 \pm 3.45 \\
37(22-42) \\
\end{array}$} & -0.074 & $\begin{array}{l}0.941 \\
(\mathrm{NS})\end{array}$ \\
\hline $\begin{array}{l}\text { Mode of delivery } \\
\text { VD } \\
\text { Instrumental } \\
\text { delivery }\end{array}$ & $\begin{array}{l}91 \\
270\end{array}$ & $\begin{array}{l}31 \\
94\end{array}$ & $\begin{array}{l}34.1 \% \\
34.8 \%\end{array}$ & $\begin{array}{l}60 \\
176\end{array}$ & $\begin{array}{l}65.9 \% \\
65.2 \%\end{array}$ & 0.017 & $\begin{array}{l}0.897 \\
\text { (NS) }\end{array}$ \\
\hline $\begin{array}{l}\text { Viability of baby } \\
\text { Non viable } \\
\text { Viable }\end{array}$ & $\begin{array}{l}15 \\
346\end{array}$ & $\begin{array}{l}5 \\
120\end{array}$ & $\begin{array}{l}33.3 \% \\
34.7 \% \\
\end{array}$ & $\begin{array}{l}10 \\
226\end{array}$ & $\begin{array}{l}66.7 \% \\
65.3 \%\end{array}$ & .012 & $\begin{array}{l}0.914 \\
\text { (NS) }\end{array}$ \\
\hline $\begin{array}{l}\text { Onset of labour } \\
\text { induced } \\
\underline{\text { spontaneous }}\end{array}$ & $\begin{array}{l}15 \\
346\end{array}$ & $\begin{array}{l}5 \\
120\end{array}$ & $\begin{array}{l}33.3 \% \\
34.7 \%\end{array}$ & $\begin{array}{l}10 \\
226\end{array}$ & $\begin{array}{l}66.7 \% \\
65.3 \%\end{array}$ & .012 & $\begin{array}{l}0.914 \\
(\mathrm{NS})\end{array}$ \\
\hline $\begin{array}{l}\frac{\text { without }}{\text { Augmentation }} \\
\text { With augmentation }\end{array}$ & $\begin{array}{l}346 \\
15\end{array}$ & $\begin{array}{l}120 \\
5\end{array}$ & $\begin{array}{l}34.7 \% \\
33.3 \%\end{array}$ & $\begin{array}{l}226 \\
10\end{array}$ & $\begin{array}{l}65.3 \% \\
66.7 \%\end{array}$ & 0.012 & $\begin{array}{l}0.914 \\
(\mathrm{NS})\end{array}$ \\
\hline $\begin{array}{l}\text { Fetal weight } \\
\underline{2500-3000 \mathrm{mg}} \\
\underline{3000-3200} \\
\underline{\text { Less than } 2500}\end{array}$ & $\begin{array}{l}312 \\
10 \\
2\end{array}$ & $\begin{array}{l}105 \\
5 \\
1\end{array}$ & $\begin{array}{l}33.7 \% \\
50 \% \\
50 \%\end{array}$ & $\begin{array}{l}207 \\
5 \\
1 \\
\end{array}$ & $\begin{array}{l}66.3 \% \\
50 \% \\
50 \%\end{array}$ & 1.552 & $\begin{array}{l}0.670 \\
(\mathrm{NS})\end{array}$ \\
\hline
\end{tabular}




\begin{tabular}{|c|c|c|c|c|c|c|c|}
\hline $\begin{array}{l}\text { Episiotomy } \\
\underline{\text { no }} \\
\text { yes }\end{array}$ & $\begin{array}{l}346 \\
15\end{array}$ & $\begin{array}{l}120 \\
5\end{array}$ & $\begin{array}{l}34.7 \% \\
33.3 \%\end{array}$ & $\begin{array}{l}226 \\
10\end{array}$ & $\begin{array}{l}65.3 \% \\
66.7 \%\end{array}$ & 0.012 & $\begin{array}{l}0.914 \\
\text { (NS) }\end{array}$ \\
\hline $\begin{array}{l}\text { Placental delivery } \\
\text { manual } \\
\text { spontaneous }\end{array}$ & $\begin{array}{l}15 \\
346\end{array}$ & $\begin{array}{l}5 \\
120\end{array}$ & $\begin{array}{l}33.3 \% \\
34.7 \%\end{array}$ & $\begin{array}{l}10 \\
226\end{array}$ & $\begin{array}{l}66.7 \% \\
65.3 \%\end{array}$ & .012 & $\begin{array}{l}0.914 \\
\text { (NS) }\end{array}$ \\
\hline $\begin{array}{l}\frac{\text { Analgesia }}{\text { sedation }} \\
\underline{\text { local }}\end{array}$ & $\begin{array}{l}15 \\
346\end{array}$ & $\begin{array}{l}5 \\
120\end{array}$ & $\begin{array}{l}33.3 \% \\
34.7 \%\end{array}$ & $\begin{array}{l}10 \\
226\end{array}$ & $\begin{array}{l}66.7 \% \\
65.3 \%\end{array}$ & .012 & $\begin{array}{l}0.914 \\
\text { (NS) }\end{array}$ \\
\hline
\end{tabular}

Table 7: Ante-partum RBCs indices in case with or without postpartum anemia.

\begin{tabular}{|c|c|c|c|c|}
\hline \multirow[t]{2}{*}{ Ante-partum RBCs indices } & \multicolumn{2}{|l|}{ Postpartum anemia } & \multirow[t]{2}{*}{ Test• } & \multirow{2}{*}{$\begin{array}{l}\text { p-value } \\
\text { (Sig.) }\end{array}$} \\
\hline & $\begin{array}{l}\text { Absent } \\
(\mathrm{N}=125)\end{array}$ & $\begin{array}{l}\text { Present } \\
(\mathrm{N}=236)\end{array}$ & & \\
\hline $\begin{array}{l}\left.\frac{\mathrm{RBCs} \text { count }\left(\times 10^{6}\right.}{\text { Mean } \pm \mathrm{SD}} \mathrm{mm}^{3}\right) \\
\text { Median (Range) }\end{array}$ & $\begin{array}{l}4.34 \pm 0.51 \\
4.30(3.20-6.50)\end{array}$ & $\begin{array}{l}3.88 \pm 0.56 \\
4(1.80-5.20)\end{array}$ & -7.050 & $\begin{array}{l}<0.001 \\
\text { (HS) }\end{array}$ \\
\hline $\begin{array}{l}\frac{\text { Hematocrit }(\%)}{\text { Mean } \pm \text { SD }} \\
\text { Median (Range) }\end{array}$ & $\begin{array}{l}35.98 \pm 3.03 \\
35.50(29.80-47.50)\end{array}$ & $\begin{array}{l}30.85 \pm 3.86 \\
30.95(15.50-41.10)\end{array}$ & -11.420 & $\begin{array}{l}<0.001 \\
\text { (HS) }\end{array}$ \\
\hline $\begin{array}{l}\frac{\text { Hemoglobin }(\mathrm{g} / \mathrm{dl})}{\text { Mean } \pm \mathrm{SD}} \\
\text { Median (Range) }\end{array}$ & $\begin{array}{l}12.39 \pm 0.91 \\
12.40(10.30-15.50)\end{array}$ & $\begin{array}{l}10.45 \pm 1.25 \\
10.60(5.40-11)\end{array}$ & -12.840 & $\begin{array}{l}<0.001 \\
\text { (HS) }\end{array}$ \\
\hline $\begin{array}{l}\frac{\mathrm{MCV}}{\mathrm{Mean}} \pm \mathrm{SD} \\
\text { Median (Range) }\end{array}$ & $\begin{array}{l}83.32 \pm 6.30 \\
84(63-100)\end{array}$ & $\begin{array}{l}79.58 \pm 7.71 \\
80.70(56-96)\end{array}$ & -4.348 & $\begin{array}{l}<0.001 \\
\text { (HS) }\end{array}$ \\
\hline $\begin{array}{l}\frac{\mathrm{MCH}}{\text { Mean }} \pm \mathrm{SD} \\
\text { Median (Range) }\end{array}$ & $\begin{array}{l}28.10 \pm 2.51 \\
28.10(20.10-34.10)\end{array}$ & $\begin{array}{l}26.45 \pm 3.20 \\
27(18.40-34.60)\end{array}$ & -4.907 & $\begin{array}{l}<0.001 \\
\text { (HS) }\end{array}$ \\
\hline $\begin{array}{l}\frac{\mathrm{MCHC}}{\text { Mean } \pm \text { SD }} \\
\text { Median (Range) }\end{array}$ & $\begin{array}{l}33.50 \pm 1.54 \\
34(27.50-36.30)\end{array}$ & $\begin{array}{l}33.14 \pm 1.81 \\
33.40(28-37.60)\end{array}$ & -2.018 & $\begin{array}{l}<0.001 \\
\text { (HS) }\end{array}$ \\
\hline $\begin{array}{l}\frac{\text { RWD }}{\text { Mean }} \pm \mathrm{SD} \\
\text { Median (Range) }\end{array}$ & $\begin{array}{l}14.42 \pm 1.80 \\
14(11.30-21)\end{array}$ & $\begin{array}{l}15.16 \pm 2.68 \\
14.50(3.10-26.90)\end{array}$ & -2.807 & $\begin{array}{l}<0.001 \\
\text { (HS) }\end{array}$ \\
\hline
\end{tabular}


Table 8: Relation between ante-partum hemorrhage and postpartum anemia.

\begin{tabular}{|c|c|c|c|c|c|c|c|c|}
\hline \multirow[t]{3}{*}{ Ante-partum hemorrhage } & \multirow{3}{*}{$\begin{array}{l}\text { Total } \\
(\mathrm{N}=361)\end{array}$} & \multicolumn{5}{|c|}{ Postpartum anemia } & \multirow[t]{3}{*}{ Test $t$} & \multirow{3}{*}{$\begin{array}{l}\text { p-value } \\
\text { (Sig.) }\end{array}$} \\
\hline & & \multicolumn{3}{|c|}{$\begin{array}{l}\text { Absent } \\
(\mathrm{N}=125)\end{array}$} & \multicolumn{2}{|c|}{$\begin{array}{l}\text { Present } \\
(\mathrm{N}=236)\end{array}$} & & \\
\hline & & \multicolumn{2}{|l|}{ No } & $\%$ & No & $\%$ & & \\
\hline $\begin{array}{l}\underline{\text { APH }} \\
\text { Absent } \\
\text { Present }\end{array}$ & $\begin{array}{l}293 \\
68\end{array}$ & \multicolumn{2}{|c|}{$\begin{array}{l}105 \\
20\end{array}$} & $\begin{array}{l}35.8 \% \\
29.4 \%\end{array}$ & $\begin{array}{l}188 \\
48\end{array}$ & $\begin{array}{l}64.2 \% \\
70.6 \%\end{array}$ & 1.006 & $\begin{array}{l}0.316 \\
(\mathrm{NS})\end{array}$ \\
\hline \multirow{3}{*}{$\begin{array}{l}\text { Estimated blood loss during } \\
\text { labour }\end{array}$} & \multirow{3}{*}{$\begin{array}{l}\text { Total } \\
(\mathrm{N}=361)\end{array}$} & \multicolumn{5}{|c|}{ Postpartum anemia } & \multirow[t]{3}{*}{ Test: } & \multirow{3}{*}{$\begin{array}{l}\text { p-value } \\
\text { (Sig.) }\end{array}$} \\
\hline & & \multicolumn{2}{|c|}{\begin{tabular}{|l|} 
Absent \\
$(\mathrm{N}=125)$
\end{tabular}} & & \multicolumn{2}{|c|}{$\begin{array}{l}\text { Present } \\
(\mathrm{N}=236)\end{array}$} & & \\
\hline & & No & $\%$ & & No & $\%$ & & \\
\hline $\begin{array}{l}200-300 \mathrm{cc}(\mathrm{VD}) \\
>300 \mathrm{cc}(\mathrm{VD}) \\
500-1000 \mathrm{cc}(\mathrm{CS}) \\
>1000 \mathrm{cc}(\mathrm{CS})\end{array}$ & $\begin{array}{l}56 \\
35 \\
184 \\
86\end{array}$ & $\begin{array}{l}23 \\
8 \\
74 \\
20\end{array}$ & $\begin{array}{l}41.1 \% \\
22.9 \% \\
40.2 \% \\
23.3 \%\end{array}$ & & \begin{tabular}{|l|}
33 \\
27 \\
110 \\
66
\end{tabular} & $\begin{array}{l}58.9 \% \\
77.1 \% \\
59.8 \% \\
76.7 \%\end{array}$ & 10.622 & $\begin{array}{l}0.014 \\
(\mathrm{~S})\end{array}$ \\
\hline
\end{tabular}

Table 9: Treatment of postpartum anemia and its outcome in the studied women.

\begin{tabular}{|c|c|c|}
\hline \multirow[t]{2}{*}{ Treatment of postpartum anemia } & \multicolumn{2}{|c|}{$\begin{array}{l}\text { Total } \\
(\mathrm{N}=236)\end{array}$} \\
\hline & No & $\%$ \\
\hline $\begin{array}{l}\text { Treated } \\
\text { Treated by oral iron } \\
\text { Treated by IV iron } \\
\text { Treated by blood transfusion }\end{array}$ & $\begin{array}{l}236 \\
168 \\
60 \\
8\end{array}$ & $\begin{array}{l}71.18 \% \\
25.42 \% \\
3.38 \%\end{array}$ \\
\hline \multirow[t]{2}{*}{ Outcome of treatment of postpartum anemia } & \multicolumn{2}{|c|}{$\begin{array}{l}\text { Total } \\
(\mathrm{N}=236)\end{array}$} \\
\hline & No & $\%$ \\
\hline $\begin{array}{l}\text { Treated } \\
\text { Improved } \\
\text { Not improved }\end{array}$ & $\begin{array}{l}236 \\
144 \\
92\end{array}$ & \begin{tabular}{|l}
61.02 \\
38.98
\end{tabular} \\
\hline
\end{tabular}

\section{Discussion}

Iron deficiency anemia (IDA) in pregnancy, i.e., prepartum anemia, is associated with premature birth, low birth weight, and small for gestational age of the newborn and is furthermore closely associated with the occurrence of anemia after delivery of a child, i.e., in the postpartum period [1]. Postpartum iron deficiency and anemia are associated with an impaired quality of life from a physical and a psychological point of view and constitutes a significant health problem both in developed and developing countries the study showed percentage of postpartum anemia is $64.3 \%$,absence of postpartum anemia is $35.7 \%$ \& showed that postpartum anemia is not affected by demographic data( age, residence) [9].

In Europe, in selected series of healthy women after normal delivery, the prevalence of anemia (hemoglobin level $<11 \mathrm{~g} / \mathrm{dL}$ ) 1 week postpartum is $14 \%$ in women who have taken iron supplements in pregnancy vs. $24 \%$ in non-supplemented women [10]. In unselected series of women who have not taken iron supplements, the prevalence of anemia (hemoglobin level $<11 \mathrm{~g} / \mathrm{dL}$ ) 48 $\mathrm{h}$ after delivery is approximately $50 \%$ [2]. In developing countries, the prevalence of postpartum anemia is Postpartum anemia is closely connected with the presence of anemia in pregnancy prior to delivery (prepartum anemia which inevitably will be aggravated after delivery due to blood losses [2]. 
The study showed percentage of types postpartum anemia

Mild normocytic normochromic anemia 42.37\% \& Mild microcytic hypochromic anemia 28.81\%\& Moderate normocytic normochromic $13.98 \%$ \& Moderate microcytic hypochromic $11.44 \% \&$ Severe normocytic normochromic $1.27 \% \&$ severe microcytic hypochromic $2.12 \%$. The study showed that cases with postpartum anemia had a mean antepartum $\mathrm{HB}$ at $10.45 \mathrm{~g} / \mathrm{dl}$ and cases without postpartum anemia had a mean antepartum $\mathrm{HB}$ at 12.39 and cases with antepartum anemia was significantly associated with postpartum anemia \& percentage of antepartum anemia was about $64.3 \%$ this percentage postpartum increased to about $65.4 \%$ and $13.6 \%$ of those with antepartum normal hemoglobin level became anemic.

In a Danish series in 1991 of iron-supplemented women after a normal singleton pregnancy and delivery, the mean hemoglobin concentration 1 -week postpartum was $12.7 \mathrm{~g} / \mathrm{dL}$ (95\% CI 10.2-14.7), whereas non-supplemented women had a mean hemoglobin of $11.8 \mathrm{~g} / \mathrm{dL}$ (95\% CI 9.7-13.9) [11].

\section{The study showed percentage of types of antepartum} anemia

mild normocytic normochromic $45.26 \%$ \& mild microcytic hypochromic $33.19 \%$ \& moderate normocytic normochromic $8.62 \%$ \& moderate microcytic hypochromic $8.62 \%$ \& severe normocytic normochromic $2.16 \%$ \& severe microcytic hypochromic $2.16 \%$.

Studies on healthy Scandinavian pregnant women without iron deficiency, singleton pregnancy, and living at sea level have shown that in late third trimester, the fifth percentile for the hemoglobin concentration is $11-11.1 \mathrm{~g} / \mathrm{dL}$ and the lower reference value of the $95 \%$ confidence interval (CI), corresponding to the 2.5 th percentile, is $10.8 \mathrm{~g} / \mathrm{dL}$ [11-14].

The study showed that antepartum hemorrhage was not significantly associated with post-partum anemia. The study showed that the more the estimated blood loss during labor the more the postpartum anemia. In a Dutch study comprising 367 women with vaginal deliveries and a low risk of postpartum hemorrhage ,blood losses within $1 \mathrm{~h}$ of delivery of placenta were measured by a gravimetric method [15].

Median (5-95 percentile) blood losses were $375 \mathrm{ml}$ (1251,500 ); losses were $\geq 500 \mathrm{ml}$ in $38 \%$ and $\geq 1,000 \mathrm{ml}$ in $10 \%$ of the women, which are higher than in studies using subjective estimates of blood losses [16]. In New South Wales blood losses $>500 \mathrm{ml}$ was observed in $13.1 \%$ of deliveries. Among deliveries in Norway in 1999-2004, severe obstetric hemorrhage $>1,500$ $\mathrm{ml}$ within $24 \mathrm{~h}$ postpartum occurred in $1.1 \%$ [17]. Postpartum hemorrhage occurs in $4-6 \%$ of all deliveries but there are considerable discrepancies between blood losses estimated by midwifes/obstetricians and true blood losses [18].

In Denmark in 2002-2005, postpartum hemorrhage (estimated blood losses $>500 \mathrm{ml}$ ) occurred in $5.1 \%$ of primipara corresponding to 3,500 deliveries per year [19]. This study showed that $71.18 \%$ treated by oral iron, $25.42 \%$ treated by IV iron , 3.38\% treated by blood transfusion \& outcome of treatment of anemia after 8 weeks $61.02 \%$ is improved \& $38.98 \%$ is not improved. In women taking $66 \mathrm{mg}$ ferrous iron/ day during pregnancy, anemia (hemoglobin level $<11 \mathrm{~g} / \mathrm{dL}$ ) at 1 week postpartum was observed in $14 \%$ vs. in $22 \%$ of women taking placebo tablets $[20,11]$. Eight weeks postpartum, anemia (hemoglobin level $<12.1 \mathrm{~g} / \mathrm{dL}$ ) was observed in $6 \%$ $8 \%$ of women who had been taking iron vs. $16 \%$ in women taking placebo $[11,20,21]$. oral iron therapy should be the first therapeutic option in women having slight-to-moderate IDA with hemoglobin levels of 9.5-12 g/dL.

They should initially be treated with ferrous iron 100-200 $\mathrm{mg}$ /day [22]. Such high doses of iron should preferably be administered between meals as a sustained release iron preparation in order to optimize absorption and reduce gastrointestinal side effects [23]. Following treatment for 2 weeks, the therapeutic response should be checked by measurement of hemoglobin. In order to keep the solution stable, iron sucrose should be dissolved in a maximum of $200 \mathrm{ml}$ isotonic saline, infused over a minimum of $30 \mathrm{~min}$; repetitive doses should be administered with a minimum of 3 -days interval [22]. At profound anemia with hemoglobin level of $<6 \mathrm{~g} / \mathrm{dl}$, blood transfusion should be considered [23, 24].

\section{References}

1. Milman N (2008) Prepartumanaemia: prevention and treatment. Ann Hemato 1 87: 949-959.

2. Bergmann RL, Richter R, Bergmann KE, Dudenhausen JW (2010) Prevalence and risk factors for early postpartum anemia. Eur J Obstet Gynecol Reprod Biol 150: 126-131.

3. Bodnar LM, Siega-Riz AM, Miller WC, Cogswell ME, McDonald T(2002) Who should be screened for postpartum anemia? An evaluation of current recommendations. Am J Epidemiol 156: 903-912.

4. World Health Organization (1999) Reduction of maternal mortality. A joint WHO/UNFPA/UNICEF/World Bank statement. World Health Organization, Geneva.

5. Potts $M$ and Campbell M (2004) Three meetings and fewer funerals: misoprostol in postpartum hemorrhage. Lancet 364: 1110-1111.

6. Mori E (2012) Study skill ii, maternity nursing 2, systematic nursing science 12: 525 .

7. Abdullahi HI, Saeed A, Imam AM, Adam I (2014) Antenatal iron and folic acid supplementation use by pregnant women in Khartoum, Sudan. BMC Research Notes 7: 498.

8. misoprostol in postpartum hemorrhage. Lancet 364: 11101111.

9. Saotome T, Murakami, et al., (2006) Recovering to make child-rearing comfortable - Support for recovery from anemia for postpartum mothers. Perinatal Care 25: 228229. 
10. Protonotariou E, Chrelias C, Kassanos D, Kapsambeli H, Trakakis E, et al. (2010) Immune response parameters during labor and early neonatal life. In Vivo 24: 117-123.

11. Milman N (2011) Anemia — still a major health problem in many parts of the world! Ann Hematol 90: 369-377.

12. Milman N, Agger OA, Nielsen OJ (1991) Iron supplementation during pregnancy. Effect on iron status markers, serum erythropoietin and human placental lactogen. A placebo controlled study in 207 Danish women. Dan Med Bull 38:471-476.

13. Milman N, Byg K-E, Graudal N, Agger AO (2000) Reference values for hemoglobin and erythrocyte indices during normal pregnancy in 206 women with and without iron supplementation. Acta Obstet Gynecol Scand 78: 89-98.

14. Milman N, Bergholdt T, Byg K-E, Eriksen L, Hvas A-M (2007) Reference intervals for haematological variables during normal pregnancy and postpartum in 433 healthy Danish women. Eur J Haematol 79: 39-46.

15. Milman N, Ibsen KK, Christensen JM (1987) Serum ferritin and iron status in mothers and newborn infants. Acta Obstet Gynecol Scand 66: 205-211.

16. Groot AN, van Roosmalen J, van Dongen PW, Borm GF (1996) A placebo-controlled trial of oral ergometrine to reduce postpartum hemorrhage. ActaObstetGynecolScand 75: 464-468.

17. Gilbert L, Porter W, Brown VA (1987) Postpartum haemorrhage a continuing problem. British Journal of Obstetrics and Gynaecology 94: 67-671.

18. Al-Zirqi I, Vangen S, Forsen L, Stray-Pedersen B (2008) Prevalence and risk factors of severe obstetric hemorrhage. BJOG 115: 1265

19. Oyelese Y and Ananth CV (2010) Postpartum hemorrhage: epidemiology, risk factors, and causes. Clin Obstet Gynecol 53:147-15654.

20. Milman N (2011) Postpartum anemia I: definition, prevalence, causes and consequences. Ann Hematol 90: 1247-1253.

21. Milman N, Bergholt T, Eriksen L, Byg K-E, Graudal N, et al. (2005) Iron prophylaxis during pregnancy-how much iron is needed? A randomised, controlled study of 20 to $80 \mathrm{mg}$ ferrous iron daily to pregnant women. ActaObstetGynecolScand 84: 238-247.

22. Breymann C, Honegger C, Holzgreve W, Surbek D (2007) Diagnostik und Therapie der Anämie in der Schwangerschaft und postpartal. Schweizerische Gesellschaft für Gynäkologie und Geburtshilfe (Quated from Milman (2012) Ann Hematol 91: 143-151

23. Nielsen JB, Ikkala E, Sölvell L, Björn-Rasmussen E, Ekenved G (1976) Absorption of iron from slow-release and rapidly disintegrating tablets - a comparative study in normal subjects, blood donors and subjects with iron deficiency anemia. Scand J Haematol Suppl 28: 89-97.

24. Beris P, Maniatis A, on behalf of the NATA working group on intravenous iron therapy(2007). Guidelines on intravenous iron supplementation in surgery and obstetrics/gynecology. Transfusion Alternatives in Transfusion Medicine 9: 29. 